of the bronchial wall of the lower lobal bronchus, which had been sutured to the carina, were removed, and the tissue was trimmed appropriately before the completion pneumonectomy (Figure $1, B$ ). The resulting flap was turned upward and used as a patch for closure of the tracheal defect (Fig. 1, C). We feared that the turned-up bronchial patch would not have sufficient blood supply from the carina through the area of the first sutures, so an omental pedicle was brought up substernally and placed to reinforce the patch and surrounding tissues.

Serial bronchoscopy revealed no complications of the suture line, and the grafted bronchus looked normal. The patient had an uneventful postoperative course for about $11 / 2$ years.

Tracheobronchoplasty occasionally entails problems in anastomosis, especially when the area of resected tissue is quite large. If in such cases dehiscence of the suture line occurs, repair of the tracheobronchial wall becomes more difficult because of the limited tissue available. Mathisen, Grillo, and Vlahakes ${ }^{1}$ reported the use of omentum in complicated thoracic problems, where it induces neovascularity and healing of the infected wound. Our patient required both of these capabilities, promotion of neovascularity of the graft patched over the defect and treatment of the intrathoracic infection caused by the dehiscence. There have been reports on a number of different patches, such as skin, pericardial tissue, and perichondrium, to close tracheal or bronchial defects. Pedicled pericardial flaps were advocated by Smolle-Jüettner and colleagues ${ }^{2}$ for closure of bronchial defects. The patients in that series, however, underwent primary operations and had no coexisting inflammation. A pericardial flap alone might not control infection around a dehiscence. Because our patient had accompanying local infection, an omental flap was necessary, regardless of the type of patch chosen. The bronchial patch was considered the best choice because it has been shown that mucociliary epithelium of the graft is preserved and regenerates after autotransplantation. ${ }^{3}$ Experimentally, tracheal autografts with omental flaps have also been proved reliable when used in pigs and dogs., ${ }^{4,5}$

To our knowledge, there have been no clinical reports demonstrating the efficacy of an autograft of the bronchus and an omental flap to patch a tracheal defect. The procedure described here may be applied in operations for cancer involving the trachea or the main bronchus but without tumor at the level of the lobar or segmental bronchi.

\section{REFERENCES}

1. Mathisen DJ, Grillo HC, Vlahakes GJ. The omentum in the management of complicated cardiovascular problems. J Thorac Cardiovase Surg 1988;95:677-84.

2. Smolle-Jüettner FM, Pinter $H$, Pongratz $G$, Ratzenhofer B, Popper H, Friehs $G$. Closure of bronchial defects using a pedicled pericardial flap. Ann Thorac Surg 1993;56:923-6.

3. Inayama $Y$, Tomiyama I, Akaike $M$, Kase $M$, Nakayama $H$, Morohosi $\mathrm{T}$, et al. Morphologic alterations and cytokinetic studies of tracheal autograft epithelium in rabbits. Ann Thorac Surg 1995;60:952-7.

4. Messineo A, Filler RM, Bahoric B, Smith C, Bahoric A. Successful tracheal autotransplantation with a vascularized omental flap. J Pediatr Surg 1991;26:1296-300.

5. Morgan E, Lima O, Goldberg M, Ferdman A, Luk SK, Cooper JD. Successful revascularization of totally ischemic bronchial autografts with omental pedicle flaps in dogs. J Thorac Cardiovasc Surg 1982;84:204-10.

\title{
PERICARDIAL PATCH AUGMENTATION OF THE TISSUE-DEFICIENT MITRAL VALVE IN COMMON ATRIOVENTRICULAR CANAL
}

Jacques A. M. van Son, MD, PhD, Richard Van Praagh, MD, Volkmar Falk, MD, and Friedrich W. Mohr, MD, Leipzig, Germany, and Boston, Mass.

Management of the mitral valve is the most critical component of the repair of common atrioventricular canal (CAVC). ${ }^{1,2}$ Patients with normal karyotype have a significantly higher reoperation rate for postoperative mitral

From the Herzzentrum, University of Leipzig, Leipzig, Germany, and the Departments of Pathology and Cardiology, Children's Hospital, Harvard Medical School, Boston, Mass.

Received for publication Dec. 5, 1995; accepted for publication Feb. 26, 1996.

J Thorac Cardiovase Surg 1996;112:1117-9

Copyright $\odot 1996$ by Mosby-Year Book, Inc.

$0022-5223 / 96 \$ 5.00+0 \quad \mathbf{1 2 / 5 4 / 7 3 1 0 1}$ valve regurgitation (as great as $50 \%$ ) than do patients with Down syndrome (as great as $10 \%$ ). ${ }^{2}$ This difference may be caused primarily by tissue scarcity, deficiency of the superior or inferior bridging leaflets, or both. On the basis of these observations, we recently developed a modified surgical technique for reconstruction of the tissue-deficient bridging leaflets of the mitral valve, with the objective of decreasing the prevalence of postoperative regurgitation in this difficult subset of cases.

In the repair of CAVC, we prefer to close the atrioventricular septal defect with a single glutaraldehyde-preserved pericardial patch. To avoid iatrogenic regurgitation of the mitral valve, the tricuspid valve, or both, it is imperative not to use too wide a patch. After assessment 


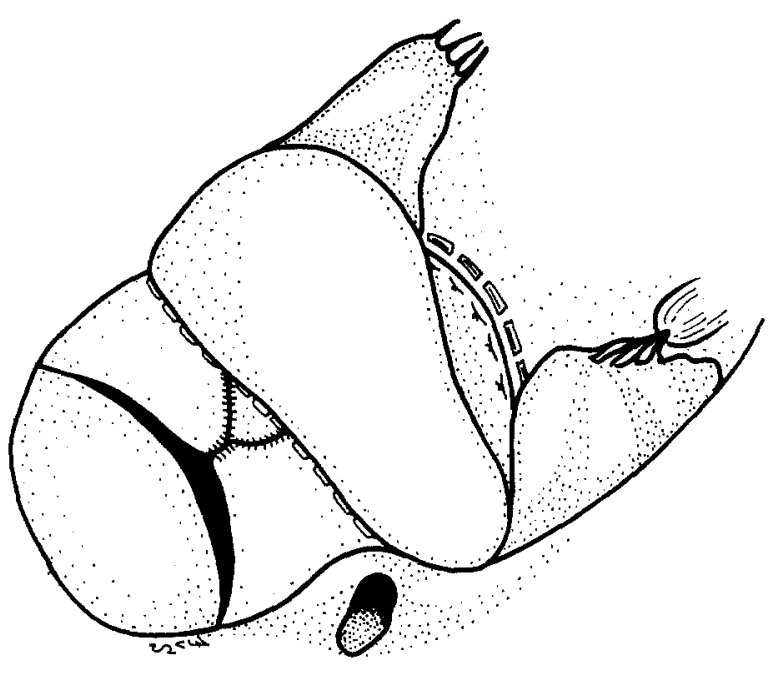

Fig. 1. Repair of complete CAVC with single glutaraldehyde-preserved pericardial patch. In case of tissue deficiency of bridging leaflets of mitral valve, free edges of bridging leaflets are approximated, untreated pericardial patch is tailored to size and configuration of defect, and this patch is anchored to atrioventricular septal patch and to edges of defect.

of valve anatomy, with particular attention to the coaptation of the bridging leaflets, a marking suture is placed to identify the point of creation of the base of the cleft in the mitral valve. Subsequently, the superior and inferior bridging leaflets are divided slightly to the right ventricular side. If sufficient valve tissue is available, the cleft separating the bridging leaflets is closed with multiple interrupted 6-0 polypropylene sutures (Prolene; Ethicon, Inc., Somerville, N.J.), up to the point at which the first set of chordal attachments is approached. The neoseptal leaflets of the tricuspid and mitral valves are anchored to the atrioventricular septal patch with multiple 5-0 braided sutures supported with small felt pledgets.

Scarcity or deficiency of leaflet tissue of the bridging leaflets may render closure of the cleft impossible. In this setting, patch augmentation of the tissue-deficient bridging leaflets may be a valuable technique. As a first step in the repair, if possible without causing undue tension, the free edges of the bridging leaflets are approximated with one or two interrupted 6-0 Prolene sutures (Fig. 1). An untreated autologous pericardial patch is tailored to the size and configuration of the defect. If the tissue defect extends up to the atrioventricular septal patch, the pericardial patch is first anchored to that patch with braided 5-0 sutures supported with felt pledgets. Subsequently, the pericardial patch is sutured to the edges of the bridging leaflets with 6-0 Prolene sutures, preferably with an interrupted suture technique. If the free edges of the bridging leaflets cannot be approximated without causing undue tension, the pericardial patch augmentation is extended up to the free edge of the neoseptal leaflet (Fig. 2). After repair of the mitral valve, the tricuspid valve, or both, ${ }^{3}$ valve function is tested by forceful injection of

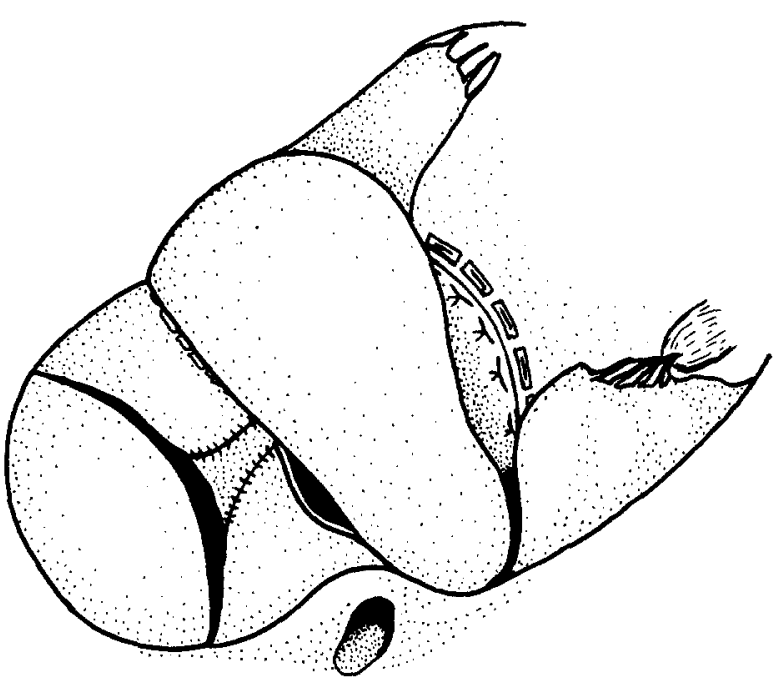

Fig. 2. If free edges of bridging leaflets cannot be approximated without causing excessive tension, pericardial patch is extended up to level of free edges of bridging leaflets.

normal saline solution into the respective ventricles. The atrial component of the atrioventricular septal defect is closed with the remaining glutaraldehyde-preserved pericardial patch. Echocardiography is routinely used to assess atrioventricular valve function and to exclude residual interatrial or interventricular communications and left ventricular outflow tract obstruction.

The reported technique was performed in five cases (median patient age 3 months, range 1 month to 3 years) of CAVC with little $(n=3)$ or no $(n=2)$ bridging of the superior leaflets and normal karyotype; four patients had moderate regurgitation of the mitral valve and three patients had associated mild left ventricular outflow tract obstruction. In four cases, the free edges of the bridging leaflets could be approximated; in the remaining case, the pericardial patch was extended up to the free edge of the bridging leaflets. In three patients, all of whom had a diminutive inferior bridging leaflet, the inferoposterior commissure was also regurgitant; a single-stitch annuloplasty resulted in a competent commissure in all three of these patients. At a median follow-up of 11 months, all patients are alive and clinically well. Echocardiography demonstrated a competent mitral valve in two patients and trivial central regurgitation in three patients.

Although the cleft between the bridging leaflets in CAVC may physiologically function as a commissure and be competent, we have major objections to the use of the term commissure in this setting. We believe that the term commissure (derived from the Latin cum and mittere, meaning "to send together"), as originally proposed by Carpentier ${ }^{1}$ and later propagated by Anderson and coworkers, ${ }^{4}$ should be reserved for a breach in the leaflet skirt of the atrioventricular valve that is supported by fan-shaped chordae tendineae inserting into a single papillary muscle group. By contrast, the chordae that are attached to the free edge of the superior and inferior 
bridging leaflets at the end of the cleft insert into two opposite papillary muscles: the chordae that control the superior bridging leaflet insert into the anterolateral papillary muscle group, whereas those controlling the inferior bridging leaflet insert into the posteromedial papillary muscle group. The tensor apparatus around the cleft does not pull the superior and inferior bridging leaflets together, but rather pulls them apart. Moreover, the cleft between the bridging leaflets is not supported by chordae.

In our experience and that of others, ${ }^{2,5}$ in patients with CAVC and normal karyotype (as opposed to patients with Down syndrome), related to the often tenuous presence of two atrioventricular valve orifices, the bridging leaflets of the atrioventricular valve may scarcely be developed, are usually displaced inferiorly, and are (partially) attached to the "scooped out" ventricular crest, resulting in a marginal area of coaptation between the bridging leaflets and potential crowding of the left ventricular outflow tract. In this setting, even mild dilatation of the common atrioventricular valve orifice or mitral valve orifice as a result of volume loading of the left ventricle (as is the case after repair of CAVC) may render the mitral valve regurgitant, especially if it has been repaired in a trileaflet fashion. Although the trileaflet atrioventricular valve concept in CAVC, as propagated by Carpentier ${ }^{l}$ and Anderson and coworkers ${ }_{2}^{4}$ is attractive from a surgical viewpoint because it allows preservation of the mitral valve in a natural configuration, we and others who have used this technique $^{2}$ have found that it can lead to unpredictable early and long-term results. Although there has been concern that bileaflet repair of the mitral valve may lead to leaflet scarring at the cleft closure site, potentially resulting in regurgitation or stenosis in the long-term, a recent clinical series in which the cleft was routinely approximated demonstrated that this type of repair holds up with time. ${ }^{6}$ At a mean 59 months after repair, 137 of 146 survivors (94\%) had only trivial or mild regurgitation of the mitral valve, and none had substantial stenosis. ${ }^{6}$ This information eliminates one of our major concerns regarding the bileaflet approach.

The technique of patch augmentation of the tissuedeficient mitral valve described here is a useful adjunct for reconstruction of atrioventricular valves in $\mathrm{CAVC}^{3}$ with encouraging medium-term results. It is important to use untreated pericardial patch, as opposed to glutaraldehyde-preserved pericardial patch, because untreated patch is more mobile and pliable. In our experience, it has superior properties as a leaflet replacement material. The technique described here may be especially valuable in the occasional case of a patient with marginal or absent coaptation of the bridging leaflets in whom closure of the cleft results in excessive tension on the cleft repair, with inherent risk of dehiscence. Another, possibly even greater benefit of this technique is that maximal height of the reconstructed neoseptal leaflet can be obtained because the bridging leaflets are approximated truly side by side, resulting in the largest possible distance between the atrioventricular septal patch and the free edge of the neoseptal leaflet and adding more surface for coaptation with the mural leaflet. In the standard technique of closure of the cleft, this height is likely to be reduced to minimize tension on the cleft repair by anchoring the central segment of the cleft to the atrioventricular septal patch, rather than closing it side by side. Finally, an additional annuloplasty (especially of the inferoposterior commissure) may be necessary to achieve complete competence of the mitral valve.

\section{REFERENCES}

1. Carpentier A. Surgical anatomy and management of the mitral component of atrioventricular canal defects. In: Anderson RH, Shinebourne EA, editors. Paediatric cardiology. Edinburgh: Churchill Livingstone, 1978:477-86.

2. Weintraub RG, Brawn WJ, Venables AW, Mee RB. Twopatch repair of complete atrioventricular septal defect in the first year of life: results and sequential assessment of atrioventricular valve function. J Thorac Cardiovasc Surg 1990;99: 320-6.

3. Falk V, van Son JA, Mohr FW. Pericardial patch augmentation of right atrioventricular valve in atrioventricular canal. Ann Thorac Surg 1996;62:288-90.

4. Anderson RH, Zuberbuhler JR, Penkoske PA, Neches WH. Of clefts, commissures, and things. J Thorac Cardiovasc Surg 1985;90:605-10.

5. Van Praagh R, Papagiannis J, Bar-El YI, Schwint OA. The heart in Down syndrome: pathologic anatomy. In: Marino B, Pueschel $\mathrm{S}$, editors. Heart disease in persons with Down syndrome. Baltimore: Paul H Brooks, 1996:69-110.

6. Bando K, Turrentine MW, Sun K, Sharp TG, Ensing GJ, Miller AP, et al: Surgical management of complete atrioventricular septal defects: a twenty-year experience. J Thorac Cardiovasc Surg 1995;110:1543-54. 